\title{
AC 2007-1060: CURRICULUM AND CONCEPT MODULE DEVELOPMENT IN RF ENGINEERING
}

\section{Robert Caverly, Villanova University}

Robert Caverly has been a faculty member at Villanova University since 1997. Prior to that he was on the faculty at the University of Massachusetts Dartmouth. He is interested in RF and microwave engineering as it pertains to RFICs and discrete control devices. 


\section{Curriculum and Concept Module Development in RF Engineering}

Introduction

The increasing number of applications students see that require wireless and other tetherless network solutions has resulted in a resurgence in Radio Frequency (RF) engineering interest at the undergraduate level. Some of these applications include not only wireless networking using such protocols as WiFi, WiMAX, and ZigBee, but also such applications as wireless sensors and RFID as well as the familiar personal communications systems. In fact, the International Technology Roadmap for Semiconductors (ITRS) shows that RF and "wireless applications may replace computers as the key driver in manufacturing" over the next 10 years. Electrical engineering departments can expect increased demand to provide well educated engineering professionals to address this increase in the need for RF and wireless engineers. As time goes on, there will also be the attendant increase in undergraduate student interest in the various RF disciplines.

Over the last several years, Villanova University, with the help of an NSF Combined Research and Curriculum Development grant, has developed a novel RF and Microwave Engineering track that introduces undergraduates to principles of this important technology. The curriculum path provides a look at the theoretical and design underpinnings and CAD tools used in a course that uses a number of design projects to reinforce the student's understanding. This paper will focus on two major points that describe our approach that addresses the education of electrical engineers in this area of contemporary interest:

- details of an undergraduate electrical engineering curriculum in RF circuit design that provides a smooth entry point either to a career path or future graduate work in this area; and

- details of a development effort into the creation of an extensive integrated series of concept modules in RF circuit design that will be made available to the general academic community in support of curriculum development areas at other universities.

Undergraduate Curriculum Track in RF and Microwave Engineering

The overall curriculum track (Figure 1) begins with the required Engineering Electromagnetics course in the junior year. This course builds upon the static electromagnetics course the students take in the freshman/sophomore year and emphasizes dynamic electromagnetics and wave theory and follows a standard course plan for a one semester course with this focus. In keeping with the curriculum track concept, an introduction to on-chip passive structures (on-chip resistors, capacitors, inductors and transformers) is introduced during the relevant discussions on the electromagnetic origins of these elements (details of this introduction can be found elsewhere ${ }^{2}$. 


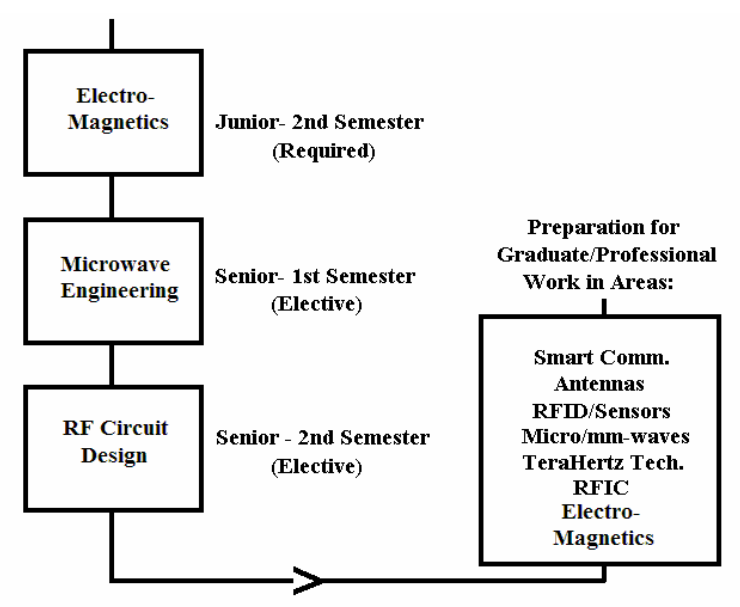

Figure 1. RF and Microwave Engineering Undergraduate curriculum flow.

The Microwave Engineering course emphasizes passive circuit design with the following high level list of topics using a widely used text ${ }^{3}$ :

- Transmission Line Theory which covers Lossless Lines, the Smith Chart and Impedance Transformation, Impedance Matching, and Lossy Lines;

- Network Theory which covers Z, Y, S and ABCD multi-port parameters, S and T two port parameters;

- Practical Transmission Lines which covers waveguides, coaxial lines, strip-style transmission lines, and transmission line filters (a brief look in this first course with more details in the follow on course);

- Microwave Power Directivity which covers power dividers and directional couplers; and

- Microwave and RF System concepts including distortion prediction using intercept points, dynamic range (linear and spurious free), system gain and noise figure and communications link budgets.

The Microwave Engineering course includes weekly laboratories where students perform basic microwave measurements and learn to use a contemporary microwave and RF CAD tool (the most recently used CAD software was Ansoft Designer SV). In addition to these weekly reinforcement exercises, the students are assigned a project to use the tools studied throughout the semester to design, simulate, layout and test a prototype microstrip low pass filter circuit based on LC ladder prototype in the nominal $1200 \mathrm{MHz}$ frequency range. The students used CAD for the design and simulation and implement the final prototype using inexpensive 1/16" thick single sided FR-4 printed circuit board and easily obtainable copper tape (1/4" and 1/2" is used). The students then use a Hewlett Packard HP-8510B Automatic Network Analyzer to measure the S-parameters of their final prototype and compare with the original set of specifications. Figure 2 shows examples of two such completed filters. 

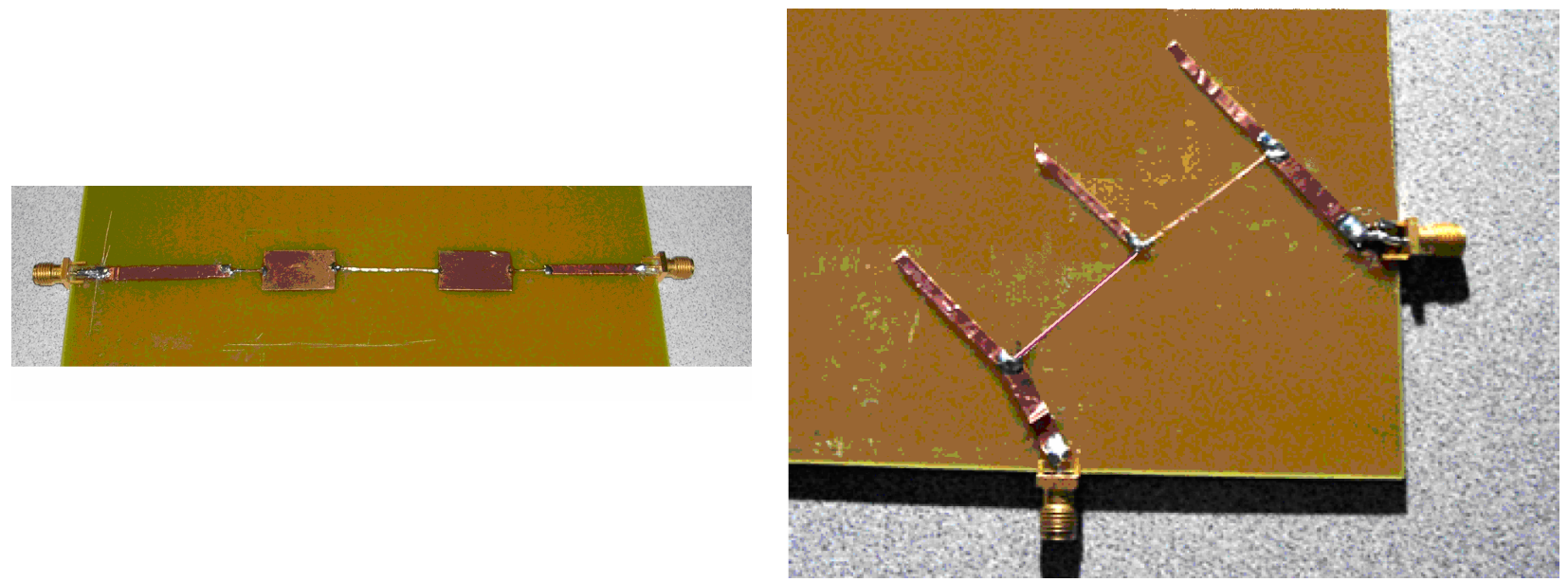

Figure 2: Examples of student-designed $1200 \mathrm{MHz}$ passive microstrip filters: stepped impedance, commensurate line low pass filters based on LC ladder prototype circuits.

This fabrication project is a key factor in students' understanding of the role of distributed element networks as opposed to the lumped element networks they have previously studied. The fact that excellent and controllable filtering characteristics can be achieved with simple copper traces on an insulating substrate generates additional discussions on tangential topics such as RF radiation from computer processor boards and unwanted trace to trace coupling on the boards. Ways to mitigate these undesired effects can also be discussed

The RF Circuit Design course is a follow-on course to the Microwave Engineering course and focuses on more active RF and microwave circuits and uses a number of hardware and CAD design projects to reinforce student understanding. In this course, the students design and fabricate and test a high frequency receiver front end and correlate the system design specifications and measurements with the material presented in this course as well as the systems concepts introduced in the previous course. The RF Circuit Design course covers the following high level list of topics

- Advanced Filtering which covers low pass, high pass, band pass and band notch filtering using LC prototypes, m-derived/constant-k filters and transmission line resonators;

- Antenna and Signal Control using primarily PIN diodes and FET-based switches;

- Mixer Design using both passive (diode, FET-based resistive) and active (biased transistor) unbalanced and balanced mixer circuit topologies;

- Amplifier Design for low power and/or low noise as well as high power (Amplifier Classes A through $\mathrm{F}$ and includes a discussion on the concept of conduction angle to define the classes);

- Oscillator Design using primarily reactance-based feedback; and

- Antenna Design with a focus on low profile printed circuit antennas.

The major course project is the design, simulation and fabrication of a $1200 \mathrm{MHz}$ receiver front end (this frequency was chosen since it is one transmitted by GPS satellites for radio location) that down converts the CW $1200 \mathrm{MHz}$ signal to an intermediate frequency (IF) of approximately $50 \mathrm{MHz}$. As the list of topics above is covered in lecture, an attendant CAD exercise and construction project is assigned as part of the classroom experience. The block 
diagram for this $1200 \mathrm{MHz}$ receiver system is shown in Figure 3. Commercial off the shelf components are used for the LNA and the diode mixer, with microstrip passive circuits used for the input band pass filter (BPF) and branch line coupler.

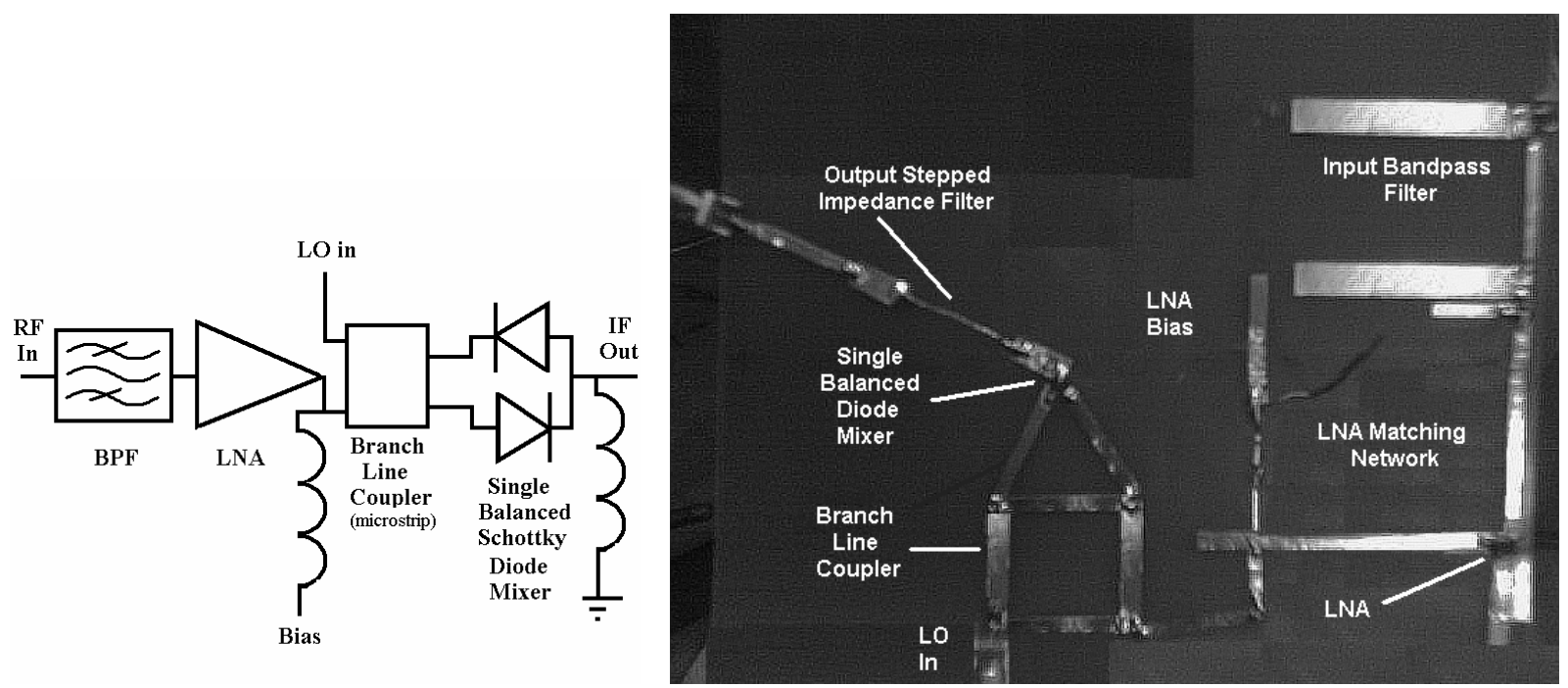

Figure 3. RF front end block diagram and a photograph of the corresponding student-designed circuit.

An accompanying antenna construction project is also performed in this final term with the antenna patterns measured at the Villanova University's compact antenna range. This antenna project is a microstrip patch antenna fabricated on 1/16" FR-4, the same substrate material used in the previous projects.

RF and Microwave Engineering Track Assessment Approach

Each course in the RF and Microwave Engineering sequence has a list of four measurable outcomes Appendix A) students are made aware of the first day of each class, and this set of outcomes is clearly marked on the course syllabi. As can be seen in the list of outcomes for the first course, students enter the RF and Microwave Engineering track with little experience in the area. With the foundational (and primarily theoretical) material covered in the first course, the primary assessment tools are traditional ones such as performance on homework, tests and written technical reports. The information from these assessments of the first course can be used as a measure of what students know and can do at the beginning of the second course. As noted in the course outcomes list in Appendix A, the remaining courses in the sequence are more oriented toward design of circuit components and system blocks (i.e., hardware). As the student progresses through the sequence, outcomes assessment becomes the answer to the question: 'Does the student design meet the performance specification described in the problem statement'. This assessment criterion is also known to the students, and has the added benefit of providing immediate feedback on their successful (or is more often the case, unsuccessful, in which case it is 'back to the drawing board') design approach; i.e., the design-fabricate-test loop has been completed. The level of student performance increases with their increasing skill set, with the final course project being a very good measure of their competence mastering $\mathrm{RF}$ and 
Microwave Engineering principles. This also shows the level of outcome achievement by exhibiting the ability to integrate various circuit and system blocks into a working and measurable RF system (such as that shown in Figure 3).

\section{Undergraduate Curriculum Concept Modules in RF and Microwave Engineering}

In 2002, a curriculum development grant from the National Science Foundation was awarded to Villanova University in the area of 'Smart Communications'. The details behind the initial organization of this program have been described in a previous paper ${ }^{4}$ Since that initial paper, an extensive array of concept modules have been developed and revised that cover foundational RF concepts that spans the range of introductory theory and design material including system concepts, advanced filter structures, amplifier, oscillator and mixer design as well as the design, construction and measurement of antennas using compact indoor antenna ranges. Each concept module contains lecture material in MSPowerPoint form with many of these lecture slides accompanied by video lectures reviewing this material. Reinforcement exercises for each module help reinforce the concepts in each module. The flow of concepts in the RF design area is shown in Figure 4

The concept module detail can be explained by using the "Introduction: System Issues" and 'RF Control' modules as examples. The 'Systems' module contains material to under elementary system concepts and starts out with a presentation on System Noise and includes material on thermal and flicker (1/f) noise, noise figure (single and cascade) and SNR. This material is followed by a discussion of the origin of system nonlinearities and starts with a discussion of general nonlinearities and ends with concepts such as distortion intercept point (single device and cascade, IP2 and IP3) and 1dB compression point. Dynamic range (linear and spurious free) can then be discussed with noise and nonlinearities as a foundation. The module ends with a detailed computation example using the previously introduced material. The 'Control' module begins with a general presentation of control concepts as on-state resistance and off-state capacitance and terminology such as insertion loss, isolation and attenuation. Specific control technologies using PIN diode, FET-based and MEMS-based control devices are then introduced with their on/off-state characteristics linked to the general control concepts described previously. Examples of tuned and untuned switches and attenuators are presented. Similar levels of detail are available in the other concept modules.

This material will be available to the general electrical engineering community by the fall of 2007. Much of this material also links to an upcoming text in CMOS RFIC circuit design 5 . The supporting material can be found by following this URL: http://rcaverly.ee.vill.edu/crcd/public.htm . 


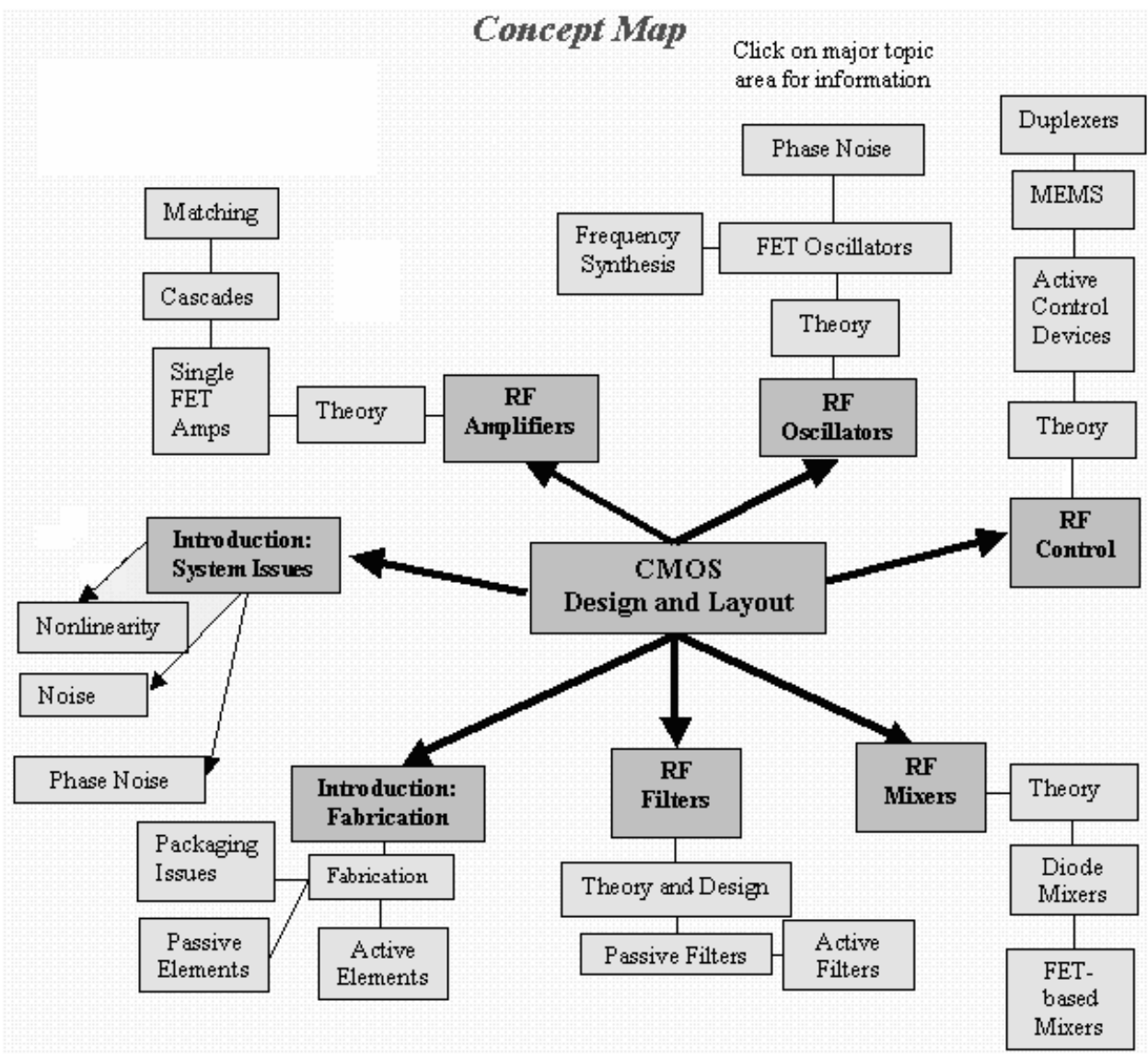

Figure 4 Concept map showing the topic flow for design of RF circuits with an emphasis on MOS circuit technology for high mixed signal integration.

\section{Acknowledgement}

This material is based on work supported by the National Science Foundation under Grant No. 0203459. A special note of thanks to Dr. Carol Weiss of VITAL (Villanova Institute for Teaching and Learning) for assistance with course and track sequence assessment.

\section{References}

[1] "2006 Update: Radio Frequency and Analog/Mixed Signal Technologies for Wireless Communications", International Technology Roadmap for Semiconductors, http://www.itrs.net/, 2006

[2] "Introducing Undergraduate Research Results in RF Microelectronics into the Undergraduate ECE Curriculum", R. Caverly, Proc. 2004 ASEE Conference, June, 2004.

[3] Microwave Engineering, $3^{\text {rd }}$ ed., D. Pozar, John Wiley and Sons, 2004.

[4] "Outcomes-based Concept Module Development for Systems for Smart Communications ", R. Caverly, M. Amin, A. Hoorfar, W. Jemison, A. Petropulu, Proc. 2003 ASEE Conference, June, 2003. 
[5] CMOS RFIC Design Principles, R. Caverly, Artech House, Norwood, MA, 2007.

Appendix A: RF and Microwave Engineering Track Outcomes on a per course basis

Measurable course outcomes: $1^{\text {st }}$ course (Junior required: Engineering Electromagnetics)

Upon completing the course, the students will have:

1. mathematically predicted the behavior of electromagnetic waves at various boundaries;

2. used modern software packages such as MATLAB and Quickfield (2-D electromagnetic field solver) to solve a variety of static and dynamic electromagnetics problems;

3. used the Smith Chart to solve impedance transformation problems; and

4. written a paper based on technical research on a current topic related to electromagnetics.

Measurable course outcomes: $2^{\text {nd }}$ course (Senior elective: Microwave Networks)

Upon completing the course, the students will have:

1. used design principles and the Smith Chart, and verify with CAD software, a variety of impedance matching structures using practical and realizable transmission lines and lumped elements.

2. used design principles and CAD software to design and simulate a variety of microwave power dividers and couplers using both waveguide and strip-style transmission lines.

3. used microwave laboratory equipment to measure fundamental microwave parameters such as SWR, insertion loss, isolation, coupling and power.

4. used modern microwave design and measurement techniques, software and instrumentation to design, simulate, fabricate and verify the operation of a passive microstrip microwave circuit.

Measurable course outcomes: $3^{\text {rd }}$ course (Senior elective: RF Circuit Design)

Upon completing the course, the students will have:

1. exhibited the ability to design RF control, amplifier and mixer circuits by fabricating a microwave front end circuit using microstrip and commercially available parts;

2. exhibited the ability to use modern microwave/RF design packages such as Ansoft Designer, MOM-based Software, Ensemble, as well as other commercial and in-house codes to solve a variety of RF system and antenna problems;

3. used the Smith Chart to solve impedance transformation, transmission line matching and amplifier design (general amplifier and LNA) problems; and

4. written several technical reports based on the design, simulation and measurements microwave/RF circuits and antennas. 